\title{
ESTIMATION OF DUCTILE FRACTURE BEHAVIOR INCORPORATING MATERIAL ANISOTROPY
}

\author{
SHIN-BEOM CHOI ${ }^{1}$, DOCK-JIN LEE ${ }^{2}$, JAE-UK JEONG ${ }^{1}$, YOON-SUK CHANG ${ }^{3 *}$, MIN-CHUL KIM ${ }^{4}$, and BONG- \\ SANG LEE ${ }^{4}$ \\ ${ }^{1}$ School of Mechanical Engineering, Sungkyunkwan University \\ 300 Chunchun-dong, Jangan-gu, Suwon, Gyeonggi-do 440-746, Republic of Korea \\ ${ }^{2}$ Nano Science and Technology, Sungkyunkwan University \\ 300 Chunchun-dong, Jangan-gu, Suwon, Gyeonggi-do 440-746, Republic of Korea \\ ${ }^{3}$ Department of Nuclear Engineering, Kyung Hee University \\ 1732 Deogyeong-daero, Giheung-gu, Yongin, Gyeonggi-do 446-701, Republic of Korea \\ ${ }^{4}$ Nuclear Material Technology Division, Korea Atomic Energy Research Institute \\ 150 Dukjin-dong, Yuseong-gu, Daejon 305-330, Republic of Korea \\ ${ }^{*}$ Corresponding author. E-mail : yschang@khu.ac.kr
}

Received October 04, 2011

Accepted for Publication February 05, 2012

Since standardized fracture test specimens cannot be easily extracted from in-service components, several alternative fracture toughness test methods have been proposed to characterize the deformation and fracture resistance of materials. One of the more promising alternatives is the local approach employing the SP(Small Punch) testing technique. However, this process has several limitations such as a lack of anisotropic yield potential and tediousness in the damage parameter calibration process. The present paper investigates estimation of ductile fracture resistance $(J-R)$ curve by $\mathrm{FE}$ (Finite Element) analyses using an anisotropic damage model and enhanced calibration procedure. In this context, specific tensile tests to quantify plastic strain ratios were carried out and SP test data were obtained from the previous research. Also, damage parameters constituting the Gurson-Tvergaard-Needleman model in conjunction with Hill's 48 yield criterion were calibrated for a typical nuclear reactor material through a genetic algorithm. Finally, the $J-R$ curve of a standard compact tension specimen was predicted by further detailed FE analyses employing the calibrated damage parameters. It showed a lower fracture resistance of the specimen material than that based on the isotropic yield criterion. Therefore, a more realistic $J-R$ curve of a reactor material can be obtained effectively from the proposed methodology by taking into account a reduced load-carrying capacity due to anisotropy.

KEYWORDS : Anisotropic Material Behavior, Genetic Algorithm, GTN(Gurson-Tvergaard-Needleman) Model, Hill's 48 Yield Criterion, Plastic Strain Ratio, Small Punch Specimen

\section{INTRODUCTION}

The structural integrity evaluation of the major components is important for safe operation of nuclear power plants. In order to examine material load-carrying capacity and degradation, various tests in use of tensile, impact and fracture toughness specimens have been tested according to relevant standards. However, the standard specimens made of archival material and the miniature specimens especially installed in the surveillance capsule of RPV(Reactor Pressure Vessel) are not enough in amount for the various tests. Therefore, alternative methodologies incorporating the $\mathrm{SP}($ Small Punch) testing technique, the ASTM E1921 test procedure[1], the ABI(Automated Ball Indentation) testing technique and so forth, respectively, were proposed.
The SP testing technique shows promise in overcoming the limitations of conventional methodology. For this technique, tiny specimens with a dimension of $10 \times 0 \times$ $0.5 \mathrm{~mm}$ are prepared, clamped in a fixture and punched as shown in Fig. 1. When the testing data is used for estimation of the ductile fracture behavior, material anisotropy should be considered to obtain reasonable results because mechanical properties of the thin and very small specimen are affected by rolling directions. Steglich et al.[2] found that the ductility of aluminum sheets decreased under applied load in the preferential orientation of the elongated second-phase particles in the rolling direction by SP test and simulation.

The commonly observed stages inside of ductile materials are void nucleation, growth of the void around the particle and coalescence of the growing void with 


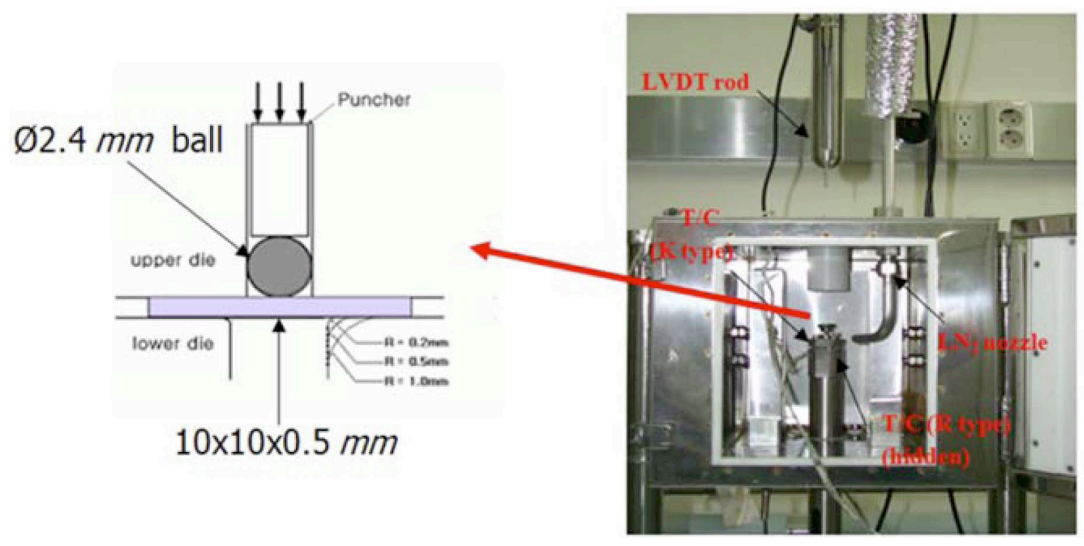

Fig. 1. Schematic of Small Punch Test Facility

adjacent ones. To deal with these micro-mechanical phenomena, the local approach employing diverse damage models has been suggested. While the GTN(GursonTverggard Needleman) model and Rousselier model have been widely used among the damage models, they did not explicitly take into account the anisotropic features of a material. To consider anisotropic material behavior, Chen et al.[3] proposed a modified GTN model in conjunction with Hill's 48 yield criterion.

In addition, it is important to calibrate damage parameters for estimating the fracture behavior because the loading type or geometry dependency may be resolved efficiently if ductile tearing can be described suitably by a constitutive equation including damage parameters. In the previous work by the authors[4], SP tests and subsequent FE(Finite Element) analyses were carried out to determine GTN damage parameters by using a trial and error method. However, a lot of time consuming effort was devoted to the calibration of damage parameters. Also, additional FE analyses were conducted to decide Rousselier damage parameters by using a GA(Genetic Algorithm)[5] which is the optimized tool based on Darwin's evolution theory [6]. Both of these researches to estimate the fracture resistance curves of nuclear reactor materials did not consider anisotropic features.

This paper investigates the estimation of ductile fracture resistance $(J-R)$ curve by $\mathrm{FE}$ analyses incorporating an anisotropic damage model and an enhanced calibration procedure. In section 2, material anisotropy is briefly reviewed in relation to ASTM E517[7] as well as Hill's 48 yield potential. Then, specific tensile tests to quantify plastic strain ratios of the material and verification of the anisotropic damage model are described in section 3 . Also, the effect of the plastic strain ratio is discussed. In section 4 , the calibration procedure by using MIGA (Multi-Island Genetic Algorithm) is introduced and, finally, $J-R$ curve is estimated by the GTN model in conjunction with Hill's 48 yield criterion.

\section{CHARACTERIZATION OF MATERIAL ANISOTROPY}

\subsection{Test Procedure to Determine Plastic Anisotropy}

ASTM E517 provides a special tensile test procedure to measure the plastic strain ratio, $r$, of a sheet metal intended for deep-drawing applications. The plastic strain ratio is a parameter that indicates ability of a sheet metal to resist thinning or thickening when subjected to either tensile or compressive force in the sheet plane. It is also a measure of plastic anisotropy and is closely related to the preferred crystallographic orientations within a polycrystalline metal. The resistance to thinning or thickening contributes to forming of geometric shapes such as cylindrical flat-bottom cups in the deep-drawing process. The $r$ value has been strained by uniaxial tensions sufficiently to induce plastic flow. This means that the ratio of the true strain occurred in the width direction $w$ perpendicular to the direction of applied stress and in the plane of the sheet to the concomitant true strain in the thickness direction $t$. Thus $r$ is numerically equal to

$$
r=\frac{\varepsilon_{w}}{\varepsilon_{t}}
$$

where, $\varepsilon_{w}$ is the width strain and $\varepsilon_{t}$ is the thickness strain, respectively, defined by

$$
\begin{gathered}
\varepsilon_{w}=\ln \left(\frac{w_{f}}{w_{0}}\right) \\
\varepsilon_{t}=\ln \left(\frac{t_{f}}{t_{0}}\right)
\end{gathered}
$$

In the above equations, $w_{0}$ is the gauge length of the transverse extensometer, $w_{\mathrm{f}}$ is the measured final gauge width, $t_{0}$ is the initial thickness and $t_{\mathrm{f}}$ is the measured final thickness. By applying the volume constant assumption, 
we can obtain

$$
\varepsilon_{t}=\ln \left(\frac{l_{0} w_{0}}{l_{f} w_{f}}\right)
$$

where, $l_{0}$ is the gauge length of the longitudinal extensometer and $l_{\mathrm{f}}$ is the measured final gauge length. Finally, the plastic strain ratio can be easily derived by the following equation:

$$
r=\frac{\ln \left(w_{0} / w_{f}\right)}{\ln \left(l_{f} w_{f} / l_{0} w_{0}\right)}
$$

\subsection{Analysis Model to Estimate Anisotropic Ductile Fracture}

The anisotropic GTN damage model affordable for a softening mechanism was adopted to simulate the fracture behaviors of ductile materials. A FORTRAN user subroutine(UMAT) coupled with a general-purpose commercial software was programmed to determine a constitutive relationship between stresses and strains. Here, the yield function is defined as

$$
\Phi^{0}=\left(\frac{q}{\sigma_{\mathrm{M}}}\right)^{2}+2 q_{1} f^{*} \cosh \left(-\frac{3}{2} \frac{q_{2} p}{\sigma_{\mathrm{M}}}\right)-\left(1+q_{3} f^{* 2}\right)=0
$$

where $q_{1}, q_{2}, q_{3}$ are calibration coefficients and $\boldsymbol{\sigma}_{m}$ is the yield strength. Comparing with the isotropic hardening $J_{2}$ plastic model, a hydrostatic stress $p$ and an effective void volume fraction $f^{*}$ have major roles to predict material behaviors such as void nucleation, growth and coalescence. An equivalent stress $q$ is generally used as the von-Mises stress in an isotropic material. Moreover, Hill's 48 stress is replaced by an equivalent stress to include effects of anisotropy into the GTN damage model.

$$
\begin{aligned}
q=F\left(\sigma_{22}-\sigma_{33}\right)^{2}+G\left(\sigma_{33}\right. & \left.-\sigma_{11}\right)^{2}+H\left(\sigma_{11}-\sigma_{22}\right)^{2} \\
& +2 L \sigma_{23}^{2}+2 M \sigma_{31}^{2}+2 N \sigma_{12}^{2}
\end{aligned}
$$

The parameters $F, G, H, L, M$ and $N$ of Hill's 48 stress are determined by the following Lankford's coefficients.

$$
\begin{aligned}
& F=\frac{r_{0}}{r_{90}\left(r_{0}+1\right)}, \quad G=\frac{1}{r_{0}+1}, \\
& H=\frac{r_{0}}{r_{0}+1}, \quad M=L=N=\frac{\left(r_{0}+r_{90}\right)\left(1+2 r_{45}\right)}{2 r_{90}\left(1+r_{0}\right)}
\end{aligned}
$$

A benchmark FE analysis by employing the single element was carried out to verify the developed UMAT code. As shown in Fig. 2, the results between the present work and Chen's work[3] agreed well, so the UMAT code can be used to simulate the ductile behavior of SP specimens with consideration of anisotropy.

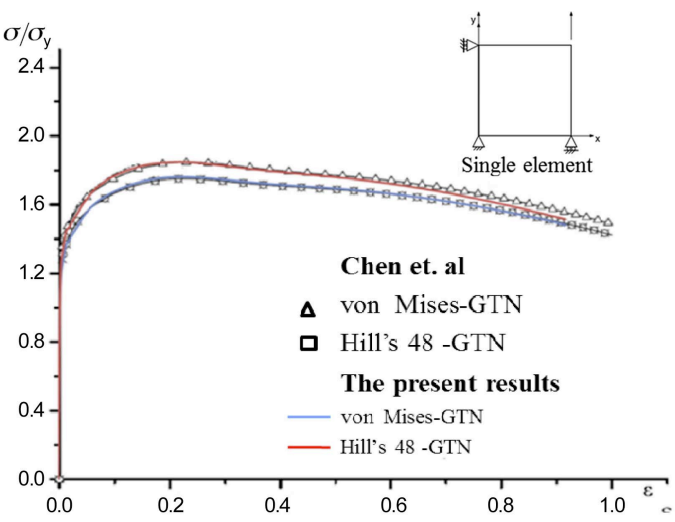

Fig. 2. Verification of UMAT Code

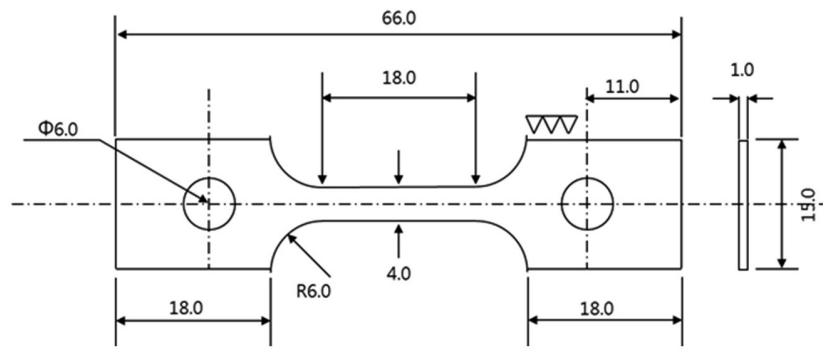

Fig. 3. Geometry of Specific Tensile Test Specimen

\section{EXPERIMENT AND SIMULATION}

\subsection{Measurement of Plastic Strain Ratio}

Specific tensile tests in three directions, such as $0^{\circ}, 45^{\circ}$ and $90^{\circ}$, were carried out on a specimen in accordance with ASTM E517 procedure, as shown in Fig. 3. The specimen material was SA508 carbon steel, which is a typical forged RPV material. As a result, different stressstrain curves were obtained and used to quantify the effect of anisotropic material behavior. The plastic strain ratios required for consideration of the anisotropic material behavior were derived from Eq. (1) or Eq. (5) and Lankford's coefficients represented in Eq. (8) based on the test data. Table 1 summarizes the resulting plastic strain ratios, which are not in agreement due to different definitions. Therefore, in this paper, all of the plastic strain ratios determined by the two equations and their average values were used to examine the effect of each directional plastic strain ratio.

\subsection{Parametric FE Analyses of Miniature Specimen}

SP simulation by adopting the FE model as shown in Fig. 4 was performed, to confirm the effect of diverse plastic strain ratios, in which refined $125 \times 125 \mu \mathrm{m}$ sized square elements were used instead of singular elements along the crack front. The GTN model combined with Hill's 48 yield criterion described in the previous section 
Table 1. Plastic Strain Ratios Based on Specific Tensile Test Data

\begin{tabular}{|c|c|c|c|c|c|c|c|c|c|c|}
\hline \multirow{2}{*}{$\begin{array}{l}\text { Direction } \\
\text { (Deg.) }\end{array}$} & \multirow{2}{*}{$l_{0}$} & \multirow{2}{*}{$w_{0}$} & \multirow{2}{*}{$t_{0}$} & \multirow{2}{*}{$l_{f}$} & \multirow{2}{*}{$w_{f}$} & \multirow{2}{*}{$t_{f}$} & \multicolumn{2}{|c|}{$r$} & \multicolumn{2}{|c|}{$r($ Avg.) } \\
\hline & & & & & & & Eq.1 & Eq. 5 & Eq.1 & Eq. 5 \\
\hline \multirow{3}{*}{0} & 12.50 & 3.99 & 1.01 & 17.98 & 3.35 & 0.87 & 1.11 & 0.93 & \multirow{3}{*}{0.97} & \multirow{3}{*}{0.87} \\
\hline & 12.46 & 3.97 & 1.01 & 17.85 & 3.37 & 0.85 & 0.92 & 0.84 & & \\
\hline & 12.45 & 3.98 & 1.01 & 17.87 & 3.38 & 0.84 & 0.90 & 0.84 & & \\
\hline \multirow{3}{*}{45} & 12.46 & 3.98 & 1.01 & 17.88 & 3.37 & 0.84 & 0.92 & 0.85 & \multirow{3}{*}{0.97} & \multirow{3}{*}{0.90} \\
\hline & 12.44 & 3.99 & 1.00 & 17.91 & 3.35 & 0.84 & 0.99 & 0.92 & & \\
\hline & 12.44 & 4.00 & 1.01 & 17.85 & 3.36 & 0.84 & 0.98 & 0.94 & & \\
\hline \multirow{3}{*}{90} & 12.46 & 3.97 & 1.02 & 17.88 & 3.36 & 0.84 & 0.84 & 0.84 & \multirow{3}{*}{0.85} & \multirow{3}{*}{0.82} \\
\hline & 12.45 & 3.98 & 1.01 & 17.79 & 3.41 & 0.84 & 0.85 & 0.78 & & \\
\hline & 12.46 & 3.98 & 1.01 & 17.84 & 3.38 & 0.83 & 0.87 & 0.84 & & \\
\hline
\end{tabular}

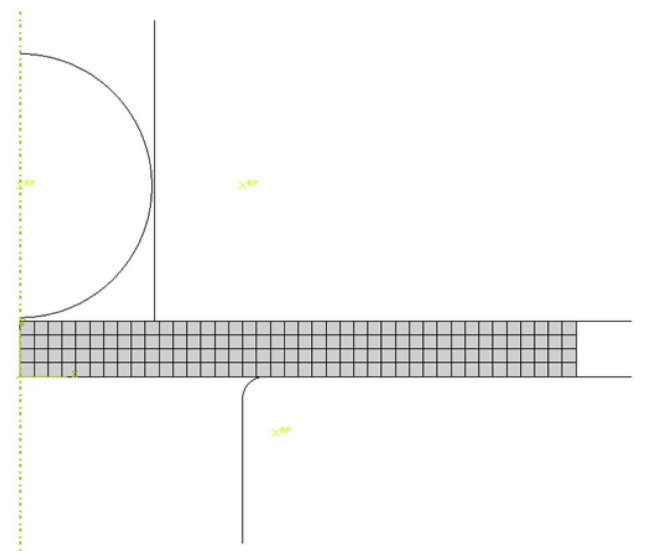

Fig. 4. FE Model of Small Punch Specimen

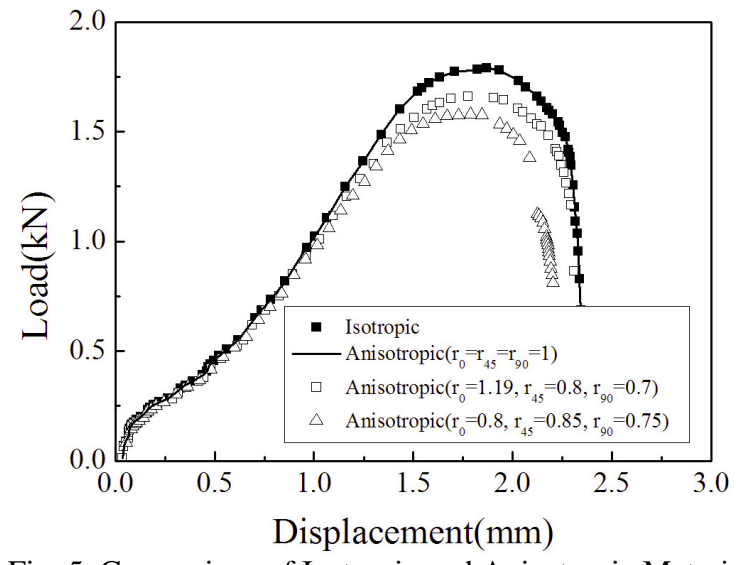

Fig. 5. Comparison of Isotropic and Anisotropic Material Behaviors had been implemented into ABAQUS/Standard[8] by using the UMAT code. Especially, elastic-plastic axi-symmetric FE analyses were carried out by using a four-node solid element(type CPE4 in ABAQUS element library). A relative sliding(finite sliding option in ABAQUS) as well as a frictional contact between the ball and specimen was taken into consideration with a measured friction coefficient $(\mu)$ of 0.25 . For this simulation, the plane of ball was set to as a rigid master surface, considering its higher stiffness and opposite portion: that is, the specimen was set as a slave surface. Since a sufficiently refined mesh was generated on the slave surface, there was no penetration of the master surface into the slave surface.

In order to investigate the effect of plastic strain ratios, preliminary FE analysis results of the SP specimen under two isotropic assumptions were compared with those under the anisotropic assumption for different $r$ values $[9,10]$. As the latter cases two sets of $r$ values such as $r_{0}=1.19, r_{45}=0.8, r_{90}=0.7[9]$ and $r_{0}=0.8, r_{45}=0.85, r_{90}=0.75$ [10] were used. As the former cases, the GTN model itself and GTN model combined with Hill's 48 yield criterion having $r_{0}=r_{45}=r_{90}=1$ were considered. As shown in Fig. 5, two isotropic assumptions led to the same loaddisplacement curves which represented the decisive evidence for validity of the UMAT code. However, the different plastic strain ratios affected the shapes as well as the maximum loads of SP load-displacement curves. It means that material anisotropy should be considered in the calibration of damage parameters and, subsequently, in the estimation of $J-R$ curves when the SP testing data is used.

To quantify the effect of plastic strain ratios of SA508 carbon steel, a total of eighteen parametric FE analyses were carried out by changing the $r$ value based on the test data summarized in Table 2. It was proven that the $r_{0}$ value was the most significant factor affecting anisotropy. Especially, as depicted in Fig. 6(a), the load- 
Table 2. Analysis Matrix to Quantify the Effect of Plastic Strain Ratios

\begin{tabular}{|c|c|c|}
\hline Case & Condition & Description \\
\hline 1 & $0^{\circ}$-Direction Stress-Strain Curve, Isotropic Material & \multirow{3}{*}{ Effects of stress-strain curves } \\
\hline 2 & $45^{\circ}$-Direction Stress-Strain Curve, Isotropic Material & \\
\hline 3 & $90^{\circ}$-Direction Stress-Strain Curve, Isotropic Material & \\
\hline 4 & $90^{\circ}$-Direction Stress-Strain Curve, Anisotropic Material $\left(r_{0}=0.97, r_{45}=0.97, r_{90}=0.85\right)$ & \multirow{2}{*}{$\begin{array}{l}\text { Effects of Eq. } 1 \text { and Eq. } 5 \\
\text { (Average } r \text { value) }\end{array}$} \\
\hline 5 & $90^{\circ}$-Direction Stress-Strain Curve, Anisotropic Material $\left(r_{0}=0.87, r_{45}=0.90, r_{90}=0.82\right)$ & \\
\hline 6 & $90^{\circ}$-Direction Stress-Strain Curve, Anisotropic Material $\left(r_{0}=1.11, r_{45}=0.92, r_{90}=0.84\right)$ & \multirow{3}{*}{$\begin{array}{l}\text { Effects of } r \text { values } \\
\text { (Eq. 1) }\end{array}$} \\
\hline 7 & $90^{\circ}$-Direction Stress-Strain Curve, Anisotropic Material $\left(r_{0}=0.92, r_{45}=0.99, r_{90}=0.85\right)$ & \\
\hline 8 & $90^{\circ}$-Direction Stress-Strain Curve, Anisotropic Material $\left(r_{0}=0.90, r_{45}=0.98, r_{90}=0.87\right)$ & \\
\hline 9 & $90^{\circ}$-Direction Stress-Strain Curve, Anisotropic Material $\left(r_{0}=1.11, r_{45}=0.85, r_{90}=0.78\right)$ & \multirow{4}{*}{$\begin{array}{l}\text { Effects of } r_{0} \text { values with fixed } r_{45} \\
\text { and } r_{90} \text { values (Eq. 5) }\end{array}$} \\
\hline 10 & $90^{\circ}$-Direction Stress-Strain Curve, Anisotropic Material $\left(r_{0}=0.93, r_{45}=0.85, r_{90}=0.78\right)$ & \\
\hline 11 & $90^{\circ}$-Direction Stress-Strain Curve, Anisotropic Material $\left(r_{0}=0.87, r_{45}=0.85, r_{90}=0.78\right)$ & \\
\hline 12 & $90^{\circ}$-Direction Stress-Strain Curve, Anisotropic Material $\left(r_{0}=0.84, r_{45}=0.85, r_{90}=0.78\right)$ & \\
\hline 13 & $90^{\circ}$-Direction Stress-Strain Curve, Anisotropic Material $\left(r_{0}=0.93, r_{45}=0.94, r_{90}=0.78\right)$ & \multirow{3}{*}{$\begin{array}{l}\text { Effects of } r_{45} \text { values with fixed } r_{0} \\
\text { and } r_{90} \text { values (Eq. 5) }\end{array}$} \\
\hline 14 & $90^{\circ}$-Direction Stress-Strain Curve, Anisotropic Material $\left(r_{0}=0.93, r_{45}=0.90, r_{90}=0.78\right)$ & \\
\hline 15 & $90^{\circ}$-Direction Stress-Strain Curve, Anisotropic Material $\left(r_{0}=0.93, r_{45}=0.85, r_{90}=0.78\right)$ & \\
\hline 16 & $90^{\circ}$-Direction Stress-Strain Curve, Anisotropic Material $\left(r_{0}=0.93, r_{45}=0.85, r_{90}=0.84\right)$ & \multirow{3}{*}{$\begin{array}{c}\text { Effect of } r_{90} \text { values with fixed } r_{0} \text { and } \\
r_{45} \text { values (Eq. 5) }\end{array}$} \\
\hline 17 & $90^{\circ}$-Direction Stress-Strain Curve, Anisotropic Material $\left(r_{0}=0.93, r_{45}=0.85, r_{90}=0.82\right)$ & \\
\hline 18 & $90^{\circ}$-Direction Stress-Strain Curve, Anisotropic Material $\left(r_{0}=0.93, r_{45}=0.85, r_{90}=0.78\right)$ & \\
\hline
\end{tabular}

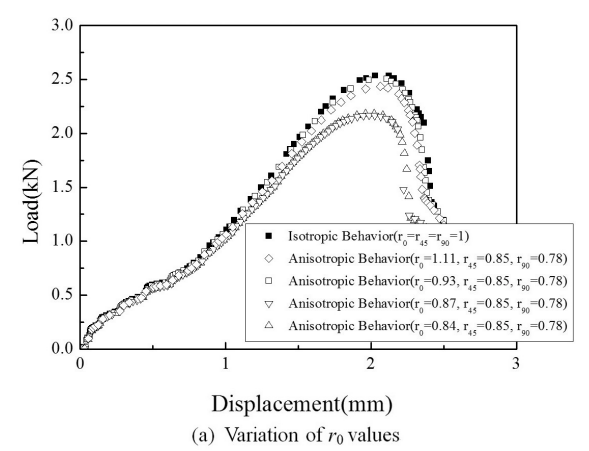

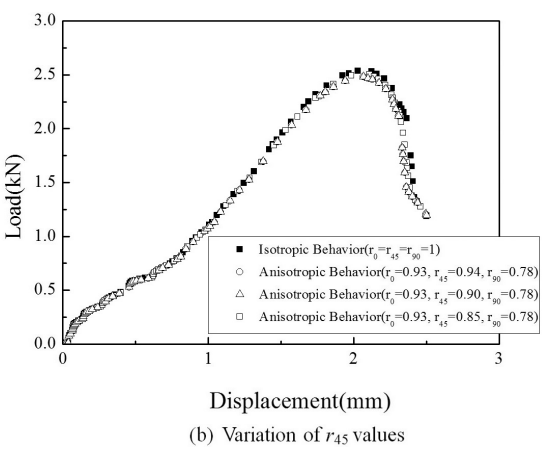

Fig. 6. Effect of Plastic Strain Ratios

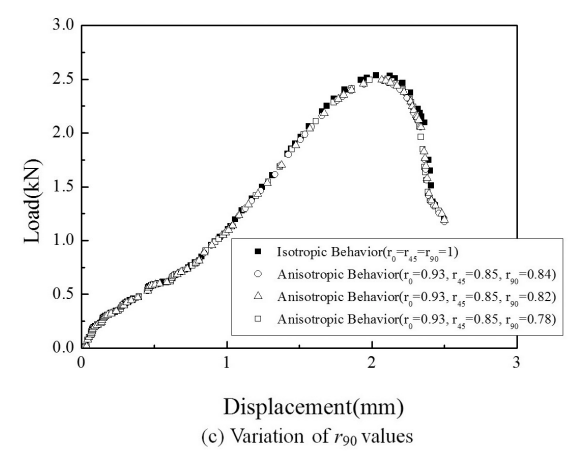

(c) Variation of $r_{90}$ values displacement curves of the anisotropic material became similar to that of isotropic material when the values of $r_{0}$ approached unity at $r_{45}=0.85$ and $r_{90}=0.78$. However, the effect of the various $r_{45}$ values at $r_{0}=0.93$ and $r_{90}=0.78$, and those of $r_{90}$ at $r_{0}=0.93$ and $r_{45}=0.85$ were negligible as shown in Fig. 6(b) and Fig. 6(c), respectively. Based on these parametric analysis results, the median set of plastic strain ratios of $r_{0}=0.95, r_{45}=0.92, r_{90}=0.87$ was selected for subsequent prediction of ductile fracture of the RPV material by considering anisotropic material behavior.

\section{ESTIMATION OF DUCTILE FRACTURE BEHAVIOR}

\subsection{Damage Parameter Calibration by using a Genetic Algorithm}

Damage parameters are generally calibrated by a trial and error method, neural network and so on, which require not only an analyzer's proficiency but also significant numerical efforts. In the author's previous work[6], a 
genetic algorithm was linked with Rousselier model under the isotropic assumption, which resulted in improvement of the calibration process. In the present research, the MIGA, among the diverse genetic algorithms, was employed for the GTN model in conjunction with Hill's 48 yield criterion to calibrate the SP test and the simulation data of SA508 carbon steel. The remarkable characteristic of MIGA is that the population in one generation is divided into several sub-populations called islands. To avoid the convergence of partially optimized results, each subpopulation maintained independency during an iteration. Before a new iteration, the exchange of individual information, called migration, was performed between subpopulations.

Fig. 7 depicts the schematic illustration of MIGA and Fig. 8 shows the calibration procedure by using the genetic algorithm combined with GTN model and Hill's 48 yield criterion. The objective function was set to minimize the error sum between the SP test and simulation by consid-

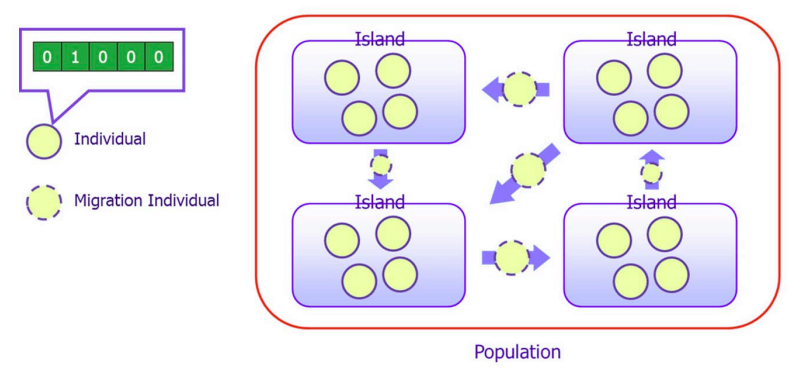

Fig. 7. Schematic Illustration of Multi-island Genetic Algorithm ering the material anisotropy. Table 3 summarizes the chemical composition of SA508 carbon steel. The relevant mechanical properties used for the calibration were $400 \mathrm{MPa}$ of yield strength and $596 \mathrm{MPa}$ of ultimate tensile strength. Fig. 9 depicts the experimental load-displacement curve [11], which is divided into 5 regions such as elastic bending, plastic bending, membrane stretching and plastic instability to facilitate correlation with the simulation curve.

For the calibration of the damage parameters, FE analyses were implemented into ABAQUS/Standard combined with the UMAT code by using the mesh of the SP specimen introduced in Fig. 4. During this process, the values of the plastic strain ratios were set to $r_{0}=0.97$, $r_{45}=0.97, r_{90}=0.85$. In addition, to reduce the number of calibration variables, the calibration coefficients of the GTN model were fixed as $q_{1}=1.5, q_{2}=1$ and $q_{3}=\mathrm{q}_{2}{ }^{2}$ according to the recommendation by Gao et al.[12]. Table 6

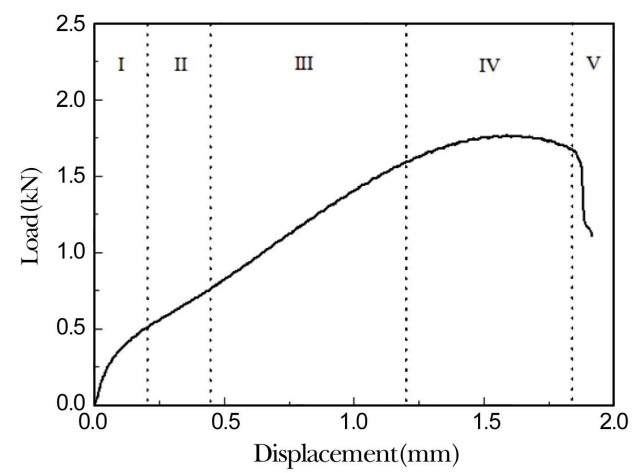

Fig. 9. Experimental Load-displacement Curve[11]

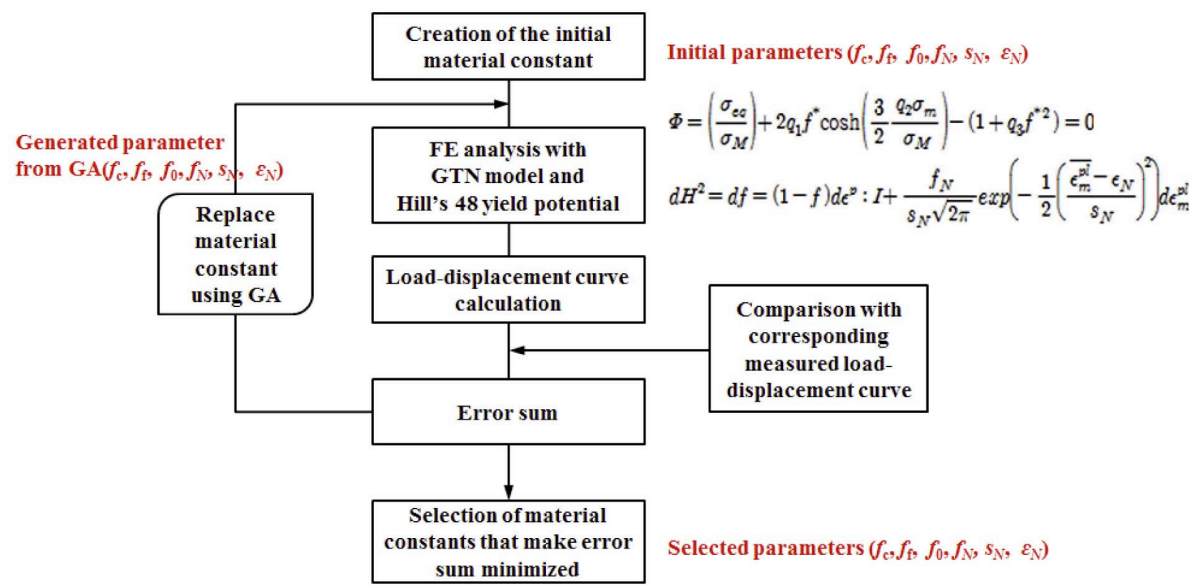

Fig. 8. Calibration Procedure by using Multi-island Genetic Algorithm Combined with GTN Model and Hill's 48 Yield Criterion

Table 3. Chemical Composition of SA508 Carbon Steel

\begin{tabular}{c|c|c|c|c|c|c|c|c|c|c}
\hline $\mathrm{C}$ & $\mathrm{Mn}$ & $\mathrm{P}$ & $\mathrm{S}$ & $\mathrm{Si}$ & $\mathrm{Ni}$ & $\mathrm{Cr}$ & $\mathrm{Mo}$ & $\mathrm{Cu}$ & $\mathrm{N}$ & $\mathrm{Al}$ \\
\hline 0.210 & 1.360 & 0.007 & 0.002 & 0.240 & 0.920 & 0.210 & 0.490 & 0.030 & - & 0.005 \\
\hline
\end{tabular}


summarizes the results of the damage parameters and Fig. 10 shows the estimated load-displacement curve of the SP specimen as well as the experimental curve. The condition of MIGA, such as size of population, number of generation, rate of crossover and rate of migration etc. that were used for the optimization of the damage parameters, is provided in Table 4. Also, Table 5 summarizes the polynomial equations that were used to calculate the error sum between the SP test and the simulation data.

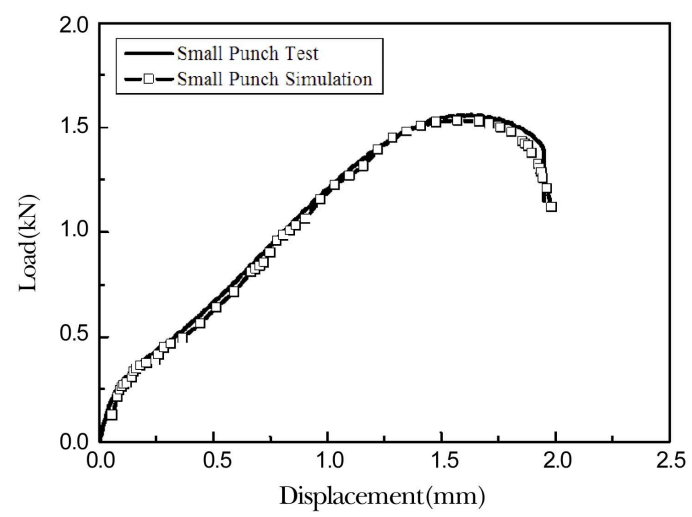

Fig. 10. Estimated Load-displacement Curve with Consideration of Anisotropic Material Behavior

Table 4. Condition of Multi-island Genetic Algorithm

\begin{tabular}{c|c|c|c}
\hline Identification & Number & Identification & Number \\
\hline Size of population & 5 & Rate of crossover & 1 \\
\hline No. of island & 5 & Rate of mutation & 0.01 \\
\hline No. of generation & 10 & Rate of migration & 0.5 \\
\hline Gene size & 32 & Interval migration & 5 \\
\hline
\end{tabular}

\subsection{J-R Curve Estimation by Considering Material Anisotropy}

A pair of analyses was conducted to predict the $J-R$ curves of the standard CT(Compact Tension) specimen. Fig. 11 depicts the FE model of 1T-CT specimen, which contains 1,496 nodes and 1,378 elements. With respect to the crack front mesh, as shown in the enlarged part of the figure, refined square elements were generated as the same size with those of SP specimen. Crack extension was identified as the size of the damage zone where the void volume fraction extended to the fracture void volume fraction due to its growth[4]. Two elastic-plastic FE analyses employing both the isotropic damage parameters

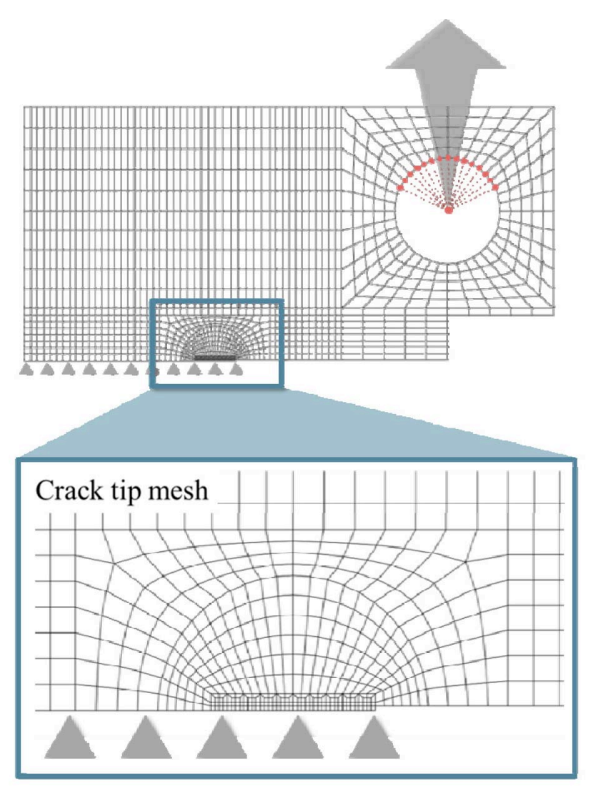

Fig. 11. FE Model of Standard CT Specimen

Table 5. Polynomial Equations to Represent Load-displacement Curve

\begin{tabular}{c|c|c|c|c}
\hline \multirow{2}{*}{ Section } & \multicolumn{4}{|c}{$\mathrm{P}=C_{1}+C_{2} \delta+C_{3} \delta^{2}+C_{4} \delta^{3}$} \\
\cline { 2 - 5 } & $C_{1}$ & $C_{2}$ & $C_{3}$ & $C_{4}$ \\
\hline$P_{\mathrm{I}}$ & 0.016 & 6.004 & -41.242 & - \\
\hline$P_{\mathrm{II}}$ & 0.253 & 0.856 & - & - \\
\hline$P_{\mathrm{III}}$ & 0.149 & 1.108 & 3.462 & 1.054 \\
\hline$P_{\mathrm{IV}}$ & 1.841 & -2.953 & - & - \\
\hline$P_{\mathrm{V}}$ & 24.658 & -12.602 & & - \\
\hline
\end{tabular}

Table 6. Calibrated Damage Parameters of GTN Model with Hill's 48 Yield Criterion

\begin{tabular}{c|c|c|c|c|c|c|c|c|c}
\hline$f_{0}$ & $f_{c}$ & $f_{f}$ & $\varepsilon_{N}$ & $f_{N}$ & $s_{N}$ & $q_{1}$ & $q_{2}$ & $q_{3}$ \\
\hline 0 & 0.043 & 0.15 & 0.43 & 0.014 & 0.09 & 1.5 & 1 & 2.25 \\
\hline
\end{tabular}




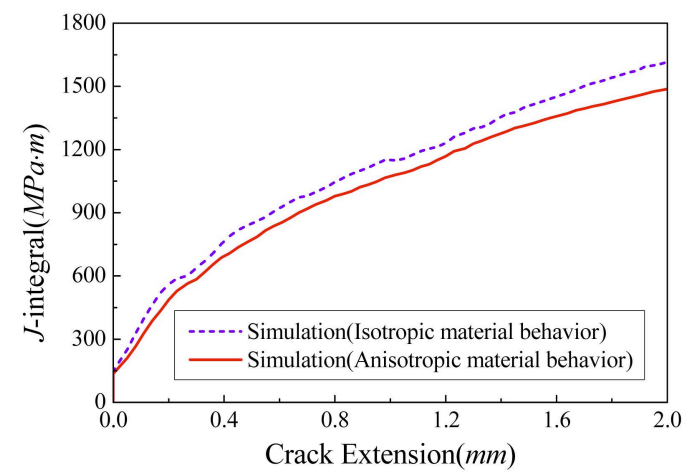

Fig. 12. Comparison of Estimated $J-R$ Curves Based on Isotropic and Anisotropic Yield Criteria

and anisotropic ones summarized in Table 6 were performed based on the deformation plasticity theory. In the present work, the $J$-integral values were extracted from the FE analysis results by using a domain integral method within ABAQUS, which showed reasonable path-independence along the crack front. Fig. 12 illustrates the comparison results in which the $J-R$ curve of the anisotropic material is lower than that of the isotropic material due to the plastic strain ratio effect. Therefore, it is anticipated that a more realistic $J-R$ curve can be obtained effectively from the proposed methodology by considering reduced load-carrying capacity due to anisotropy.

\section{CONCLUSIONS}

In this paper, the ductile fracture behavior of a typical RPV material was investigated through a series of FE analyses incorporating the anisotropic damage model and enhanced calibration procedure. The key results and findings are summarized as follows:

(1) Specific tensile tests were performed to decide the anisotropic measure in accordance with ASTM E517. The effect of diverse directional plastic strain ratios on load-displacement curves was examined and $r_{0}$ was found to be the most influential parameter.

(2) A UMAT code was developed for the GTN model in conjunction with Hill's 48 yield criterion and MIGA was employed to efficiently calibrate the relevant damage parameters of a tiny SP specimen.

(3) A pair of analyses, employing both the isotropic damage parameters and anisotropic ones, were conducted to predict the ductile fracture behavior of a standard CT specimen. The comparison of the results showed that the $J-R$ curve of the anisotropic material was lower than that of the isotropic material due to the plastic strain ratio effect.

\section{ACKNOWLEDGEMENT}

We would like to express deep appreciation to the late Professor Young-Jin Kim, who not only provided helpful suggestion but also guided this research to a successful completion.

\section{REFERENCES}

[1] American Society of Testing Materials, "Standard test method for determination of reference temperature, $\mathrm{T}_{0}$, for ferritic steel in the transition range," E1921-05, 2005.

[2] D. Steglich, W. Brocks, J. Heerens and T. Pardoen," Anisotropic ductile fracture of $\mathrm{Al} 2024$ alloys," Engineering Fracture Mechanics, Vol. 75, pp. 3692-3706, 2008.

[ 3 ] Z. Chen and D. Xianghuai, "The GTN damage model based on Hill' 48 anisotropic yield criterion and its application in sheet metal forming," Computational Materials Science, Vol. 44, pp. 1013-1021, 2009.

[4] Y.S. Chang, J.M. Kim, J.B. Choi, Y.J. Kim, M.C. Kim and B.S. Lee, "Derivation of ductile fracture resistance by use of small punch specimens," Engineering Fracture Mechanics, Vol. 75, pp. 3413-3427, 2008.

[5] D.E. Goldberg, "Genetic algorithm in search, operation and machine learning," Addison-Wesley Publishing Co. Inc., New York, USA, 1989.

[6] J.U. Jeong, S.B. Choi, Y.S. Chang, J.B. Choi, Y.J. Kim, M.C. Kim and B.S. Lee, "Calibration of damage parameters for Y5 and G91 steels in use of genetic algorithm," 20th International Conference on Structural Mechanics in Reactor Technology, 2009.

[ 7 ] American Society of Testing Materials, "Standard test method for plastic strain ratio $r$ for sheet metal," E517-00, 2006.

[ 8 ] Dassault Systems, "ABAQUS Version 6.4 user's manual," 2004.

[9] A. Chamekh, H. Belhadjsalah, R. Hambli and A. Gahbiche, "Inverse identification using the bulge test and artificial neural networks," Journal of Materials Processing Technology," Vol. 177, pp. 307-310, 2006.

[10] www.bssa.org.uk/cms/file.

[11] Sungkyunkwan University, "Analysis technology of local fracture behavior based on finite element method," KAERI /CM-999/2006, 2006.

[12] X. Gao, J. Faleskog, C.F. Shih and R.H. Dodds, "Ductile tearing in part-through cracks: Experiments and cellmodeling predictions," Engineering Fracture Mechanics, Vol. 59, pp. 61-77, 1998. 